\title{
HIV: to vaccinate or not to vaccinate?
}

\author{
Gordon Ada, Bob Blanden and Arno Mullbacher
}

\section{"Improbability of effective vaccination against human immunodeficiency virus . . .", declares the title of a new paper by Dr Albert Sabin. But three immunologists see flaws in his argument.}

THE task of developing a vaccine against the human immunodeficiency virus (HIV) is both urgent and difficult. Is it likely to succeed? Dr Albert Sabin, an eminent virologist, thinks not, and last month $^{1}$ set out his reasons for that belief. He was quite right to do so, for he raises some serious scientific issues. But in our view such pessimism is premature, and his doubts can be largely allayed on immunological grounds.

Last year, Sabin pointed out ${ }^{2}$ that one potential difficulty was whether any vaccine against HIV would protect a person from infected cells in semen. In his latest paper $^{1}$ (the full title of which is "Improbability of effective vaccination against human immunodeficiency virus because of its intracellular transmission and rectal port of entry") he reiterates this message; he quotes several unsuccessful protection experiments with cells infected with simian immunodeficiency virus (SIV) and comments upon an experiment in which vaccinated chimpanzees successfully withstood a challenge of HIV-infected blood cells ${ }^{3}$. He now draws attention to the high content of virus and of HIV-infected cells in some samples of semen. But in particular Sabin has reservations whether vaccination could be effective against cells containing "cytoplasmic pro-virus" and/or chromosomally integrated HIV complementary DNA, because it was thought that such cells would not express viral products at their surface.

In the vast majority of cases, however, the major histocompatibility complex (MHC) antigens at the cell surface would be foreign (allogeneic) to the recipient. Some of the lympho-myeloid cells would be quite effective as stimulators of a rapid host-versus-graft response, whether or not they were infected, and the rejection (destruction) of all semen-derived cells would follow. One way in which a response by the normal host to allogenic lympho-myeloid cells could be avoided would be a drastic down-regulation of MHC antigen expression or other co-stimulatory activities in infected cells. This does not occur to a substantial extent in productively HIV-infected lymphocytes or monocytes, and so is even less likely to occur in nonproductively infected cells. Semen is also reported to have immunosuppressive properties, but these would need to be very powerful to prevent an

\section{allogeneic reaction in vivo.}

Cells containing endogenous C-type viruses have been shown, perhaps unexpectedly, to express viral peptides associated with MHC molecules ${ }^{4}$. By analogy, if the HIV cDNA in live or dead semen cells were to be transferred undamaged in some novel way to live host cells, as Sabin suggests, expression of viral peptides could also occur. If so, the 'transfected' host cells would be recognized by cytotoxic $\mathrm{T}$ lymphocytes (CTLs) in the vaccinated host. Sabin rightly emphasizes the importance of cell-mediated responses in controlling viral replication.

There are other possibilities. Might some cells in the semen escape immunosurveillance by quickly reaching immunoprivileged sites in the recipient? We are unaware of any evidence that this is likely to occur. Might a small proportion of the donor's infected cells initiate a graft-versus-host response, so becoming activated and expressing viral antigens? Anti-HIV antibody generated following vaccination should deal with such cells, either by antibody-dependent cellular cytotoxicity or C-dependent lysis. Cells in the donated semen already expressing viral antigen could fuse with host cells (gp120/CD4 interaction), and thus be recognized by both class I MHCrestricted CTLs resulting from the vaccination and alloreactive CTLs. Although there is uncertainty about the likelihood of some theoretical possibilities, we believe that these particular concerns are not a serious barrier to vaccine development and use.

Sabin puts stress on another point, the hazards of receptive anal intercourse, though the extent to which these hazards also apply in heterosexual vaginal intercourse is not clear. The content of infectious virus-infected cells in the semen of asymptomatic, infected men varies greatly, from high levels (quoted by Sabin) to levels undetected by the polymerase chain reaction ${ }^{5}$. In the absence of other sexually transmitted diseases, the efficacy of transmission of HIV infection by sexual intercourse, including receptive anal intercourse, and needle sharing, is very low -1 per cent or less ${ }^{6}$. Such values, together with a related finding (that the initial viraemia occurring after infection seems usually to reflect the replication of virus of a single antigenic specificity ${ }^{7,8}$ ) are consistent with infection being initiated by a low dose of infectious virus in many cases.

A further issue raised by Sabin ${ }^{1}$ is the failure of antibody to neutralize virus during an infection. However, the very fact that escape mutants are found so frequently during an infection indicates that antibody of the appropriate specificity is effective. This view is supported by the finding ${ }^{9}$ that a monoclonal antibody against the HIV gp120 V3 loop protected two chimpanzees from a large dose (75 chimpanzee infectious doses) of virus whether the antibody was administered before or after the challenge. Unquestionably, more needs to be found out about the effectiveness of secretory immunoglobulin A in preventing infection and the feasibility of inducing a persistent response in the rectum and in the female reproductive tract. There is a recent report that monkeys previously vaccinated by parenteral administration against SIV, withstood first a challenge by SIV given parenterally and then a subsequent challenge of SIV delivered via the rectum ${ }^{10}$.

There is a long way to go before highly effective anti-HIV vaccines are formulated. Yet, there is reason to believe that 'first-generation' vaccines, even though they may not possess all of the desirable properties, could prevent infection in a proportion of recipients, and much could be learnt to facilitate future vaccine development. An answer will not be available until the best candidates or combinations of candidates, either already under phase 1 trials or about to undergo such trials, meet certain guidelines (including safety) and can then be tested in appropriately designed and executed phase 3 trials. To delay or not perform such trials for the reasons proposed by Dr Sabin would be disastrous for the increasing numbers of people exposed to the risk of infection. $\square$

Gordon Ada, Bob Blanden and Arno Mullbacher are in The John Curtin School of Medical Research, Australian National University, Canberra, ACT 2601, Australia.

\footnotetext{
1. Sabin, A. B. Proc. natn. Acad. Sci. U.S.A. 89, 88528855 (1992)

2. Sabin, A. B. Science 251, 1161 (1991).

3. Fulz, P. N et al Science 256, 1687-1690 (1992)

4. Janeway, C. A. 8th Int. Cong. Immun., Budapest, 1992

5. Krieger, J. N. et al. J. inf. Dis. 163, 386-388 (1992)

6. Blattner, W. A. FASEB J. 5, 2340-2348 (1991).

7. Wolfs, T. F. W. et al. Virology 185, 195-205 (1991)

8. Wolfs, T. F. W. et al. Virology 189, 103-110 (1992)

9. Emini, E A et al Nature 355, 728-730 (1922)

10. Cranage, M. P. et al, Lancet 339. 273-274 (1992)
} 